\title{
Developing the Pulsed Fission-Fusion (PuFF) Engine
}

\author{
Robert B. Adams, Ph.D. ${ }^{1}$ \\ National Aeronautics and Space Administration, MSFC, AL 35812 \\ Jason Cassibry, Ph. D. ${ }^{2}$ \\ University of Alabama in Huntsville, Huntsville, AL 35812 \\ David Bradley ${ }^{3}$ \\ Yetispace, Inc., Huntsville, AL 35812 \\ Leo Fabisinski ${ }^{4}$ \\ International Space Systems, Inc., Huntsville, AL 35812 \\ and \\ Geoffrey Statham, D. Phil. ${ }^{5}$ \\ ERC, Inc.
}

\begin{abstract}
In September 2013 the NASA Innovative Advanced Concept (NIAC) organization awarded a phase I contract to the PuFF team. Our phase 1 proposal researched a pulsed fission-fusion propulsion system that compressed a target of deuterium (D) and tritium (T) as a mixture in a column, surrounded concentrically by Uranium. The target is surrounded by liquid lithium. A high power current would flow down the liquid lithium and the resulting Lorentz force would compress the column by roughly a factor of 10 . The compressed column would reach criticality and a combination of fission and fusion reactions would occur. Our Phase I results, summarized herein, review our estimates of engine and vehicle performance, our work to date to model the fission-fusion reaction, and our initial efforts in experimental analysis.
\end{abstract}

\section{Nomenclature}

$=$ Target Surface Area

$=$ Magnetic Field pressure

$=$ Electric Field

$=$ fractional deposition of alpha particles

$=$ smoothing length

$=$ Magnetic Field

$=$ current density

$=$ thermal conductivity, Boltzmann's constant

$=$ mass

$=$ number density

$=$ Pressure

$=$ fusion reaction energy

$=$ thermal equilibrium term

$=$ position

$=$ friction tensor

\footnotetext{
${ }^{1}$ Aerospace Engineer, ER24/Advanced Propulsion and Technology, Associate Fellow.

${ }^{2}$ Associate Professor, Tech Hall, Room TH S232, Propulsion Research Center, University of AL in Huntsville, Huntsville, AL 35899, Lifetime Senior Member.

${ }^{3}$ Aerospace Engineer, Yetispace, Inc., 7902 Logan Dr. Huntsville, Al, 35802.

${ }^{4}$ Aerospace Engineer, ISSI, Inc., Huntsville, AL, 35806.

${ }^{5}$ Research Scientist, ERC, Inc., Huntsville, AL, 35806.
} 


$\begin{array}{ll}t & =\text { time } \\ T & =\text { Temperature } \\ u & =\text { velocity } \\ V & =\text { Volume } \\ W & =\text { kernel } \\ Z & =\text { ion charge } \\ \varepsilon & =\text { permittivity } \\ \sigma & =\text { conductivity } \\ \pi & =\text { viscous stress tensor } \\ \mu & =\text { magnetic permeability } \\ \rho & =\text { density } \\ \langle\sigma v> & =\text { velocity averaged fusion cross section }\end{array}$

\section{Introduction}

$\mathrm{T}$ HE PuFF concept uses a Z-pinch configuration but uses a fission reaction to boost the fusion process. The column contains a central cylinder of D-T plasma, surrounded by a U-238 cylindrical sheath, which is in turn surrounded by a lithium cylindrical sheath. The D-T mixture is compressed until a limited number of fusion reactions take place (well below 'break-even' energy). The resulting fast thermonuclear neutrons bombard the surrounding U-238 and induce fission which in turn increases the fusion yield of the D-T core.

There is considerable synergy inherent in this concept. D-T are the easiest fusion fuels to ignite, but are the least desirable because most of the energy release is in the form of high-speed neutrons, which are of little use for power generation or thrust and damage the propulsive structure. In the proposed concept the neutrons from both fission and fusion reactions are well reflected and moderated by the lithium liner. Also the neutron release from fusion will result in more complete consumption of the fissile fuel, sustaining energy release. The sustained release extends the compression of the fusion reactants, yielding more fusion reactions. And of course more fusion release means more neutrons for more fissile consumption. This fortuitous cycle means that the fission event is not simply used to ignite fusion, but also helps achieve a more complete burn-up of the fission and fusion fuels. This synergy has been observed in the development of other fission-fusion devices.

Using fission-fusion synergy means that ignition may be achieved with lower energy input through the Z-pinch. Lower energy input will translate into smaller capacitor banks and a smaller overall propulsion system. The proposal team hopes to develop a considerably smaller hybrid propulsion system. A smaller system would be considerably less expensive to develop and would be applicable to a wider range of NASA missions. Also, while the concept of the fission-fusion hybrid is rooted in weapons design, our concept has built in non-proliferation features. Even with the reductions in size our team hopes to achieve with this concept, it will still be far too large to ever be a terrorist weapon. Finally the propulsion system has a built in method to vary specific impulse. Trading specific impulse for thrust is desirable when optimizing a trajectory. By varying the thickness of the lithium liner our concept can easily increase thrust (at the expense of specific impulse)..

\section{Background}

The idea of combining fission and fusion, so that the former process assists the latter, is well established - most obviously in the field of nuclear weapons; however, it has seen relatively little application in the field of space propulsion. The most obvious exception was Project Orion, during the late 1950s and early 1960s, which proposed to expel thermonuclear bombs from the base of a very large vehicle and detonate them a short distance away. The resulting blast wave would then impact a 'pusher' plate at the rear of the vehicle, and thrust would be transmitted to the vehicle by means of a set of shock absorbers.

Project Orion has been well-documented - see, for example, Ref. ${ }^{\mathrm{i}}$ - and was intended to use actual thermonuclear weapons, rather than a wholly unique design, so it will not be considered further here; however, there are several more recent concepts - not utilizing weapons hardware - which are of more relevance to PuFF. Before reviewing them it is instructive to establish a simple taxonomy of fission-fusion hybrid systems as follows. 


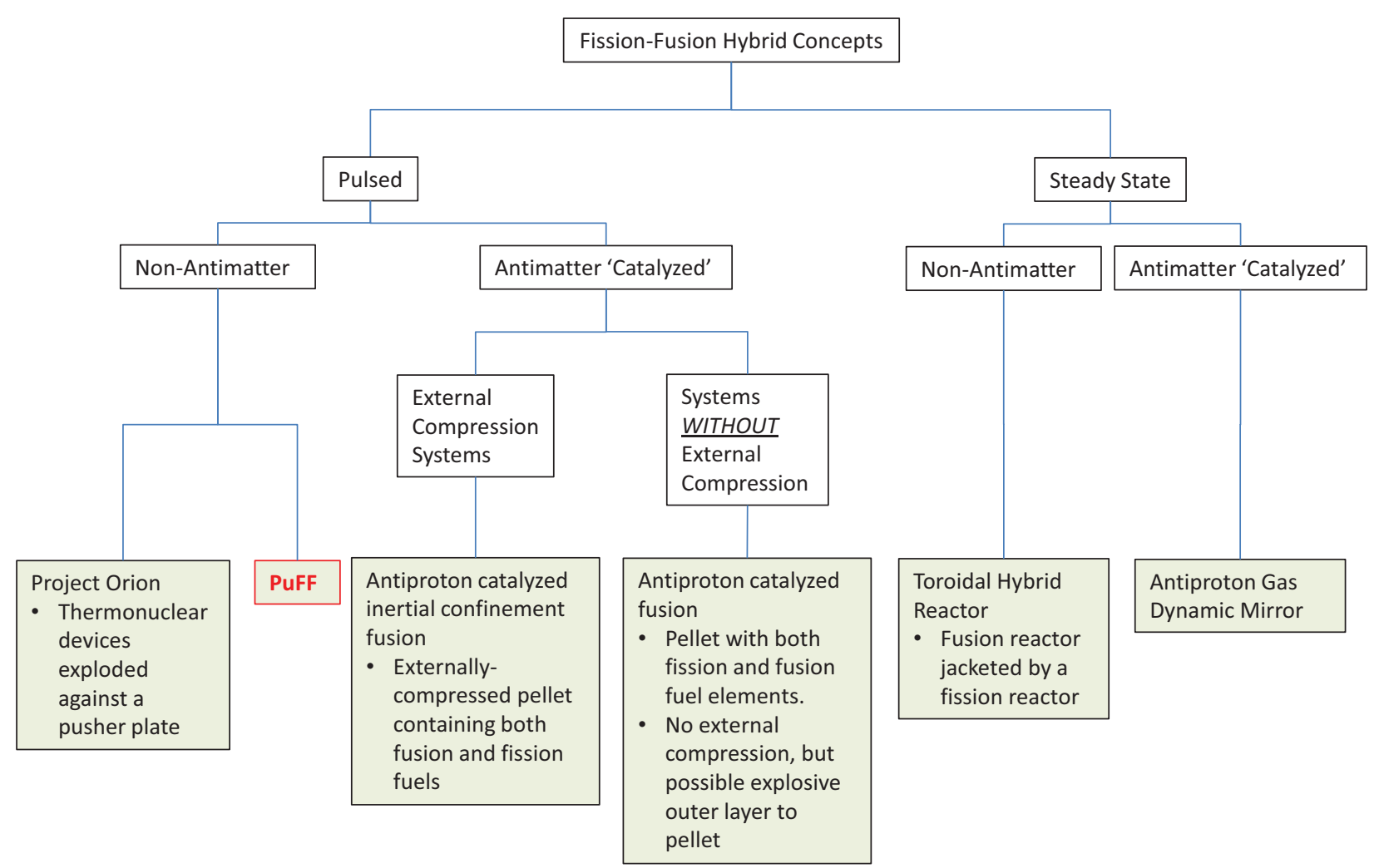

Figure 1. Taxonomy of Fission-Fusion Hybrid Propulsion Concepts

\section{Integrated Steady State Model}

In the theoretical analysis, we are modeling fission and fusion energy released in so-called 'targets'. These consist of a magnetized plasma surrounded by layers of lithium and either uranium or thorium. To assess feasibility we need to determine the density, temperature, and thickness of each of these layers in order to find the regions of parameter space in which the system leads to a net heating of the target. The power balance is

$\frac{\mathrm{dE}}{\mathrm{dt}}=\mathrm{P} \frac{\mathrm{dV}}{\mathrm{dt}}+\mathrm{f}_{\alpha} \mathrm{Qn}^{2}\langle\sigma v\rangle \mathrm{V}-\mathrm{P}_{\text {brems }}-\mathrm{P}_{\text {sync }}-\mathrm{Ak} \nabla \mathrm{T}$

where $\mathrm{P}$ is the pressure, $\mathrm{V}$ is the target volume, $\mathrm{f}_{\alpha}$ is the fractional deposition of alpha particles, $\mathrm{Q}$ is the fusion reaction energy, $\mathrm{n}$ is the particle number density, $\langle\sigma v\rangle$ is the velocity averaged cross section for the fusion reaction, $\mathrm{A}$ is the target surface area, $\mathrm{k}$ is the thermal conductivity, and $\mathrm{T}$ is the temperature. $\mathrm{P}_{\text {brems }}$ represents Bremsstrahlung radiation loss given by

$\mathrm{P}_{\text {brems }}=1.44 \mathrm{e}^{-40} \mathrm{n}^{2} \mathrm{~T}^{\frac{1}{2}} \mathrm{~V}$

and $\mathrm{P}_{\text {sync }}$ represents synchrotron radiation loss given by,

$\mathrm{P}_{\text {sync }}=5.342 \mathrm{e}^{-24} \mathrm{nB}^{2} \mathrm{~T}\left(1+\frac{\mathrm{T}}{2.367 \mathrm{e}^{9}}\right) \mathrm{V}$

The first term $(\mathrm{PdV} / \mathrm{dt})$ represents the work done by the compression of the liner on the target. The second term is the power from fusion reactions. The last term is the thermal conduction loss.

Briefly, we assume a cylinder of plasma with a DD or DT mixture, and vary the temperature and density. We investigated the effects of ${ }^{235} \mathrm{U},{ }^{238} \mathrm{U},{ }^{232} \mathrm{Th}$, and ${ }^{6} \mathrm{Li}$ liners on DT gas targets with a magnetic field comparable to what would be generated by a 2 MA Z-pinch produced in the Charger 1 facility. For the DT plasma, we assumed all neutrons pass through the surrounding liner with an energy of $14.1 \mathrm{MeV}$. The high $\mathrm{Z}$ materials all yield reactions with $\sim 160 \mathrm{MeV}$ of fission fragments per reaction, while the lithium produces $4.784 \mathrm{MeV}$ per reaction. The neutron fission cross sections were taken from the Evaluated Nuclear Data File (Ref. ${ }^{i i}$ ). 

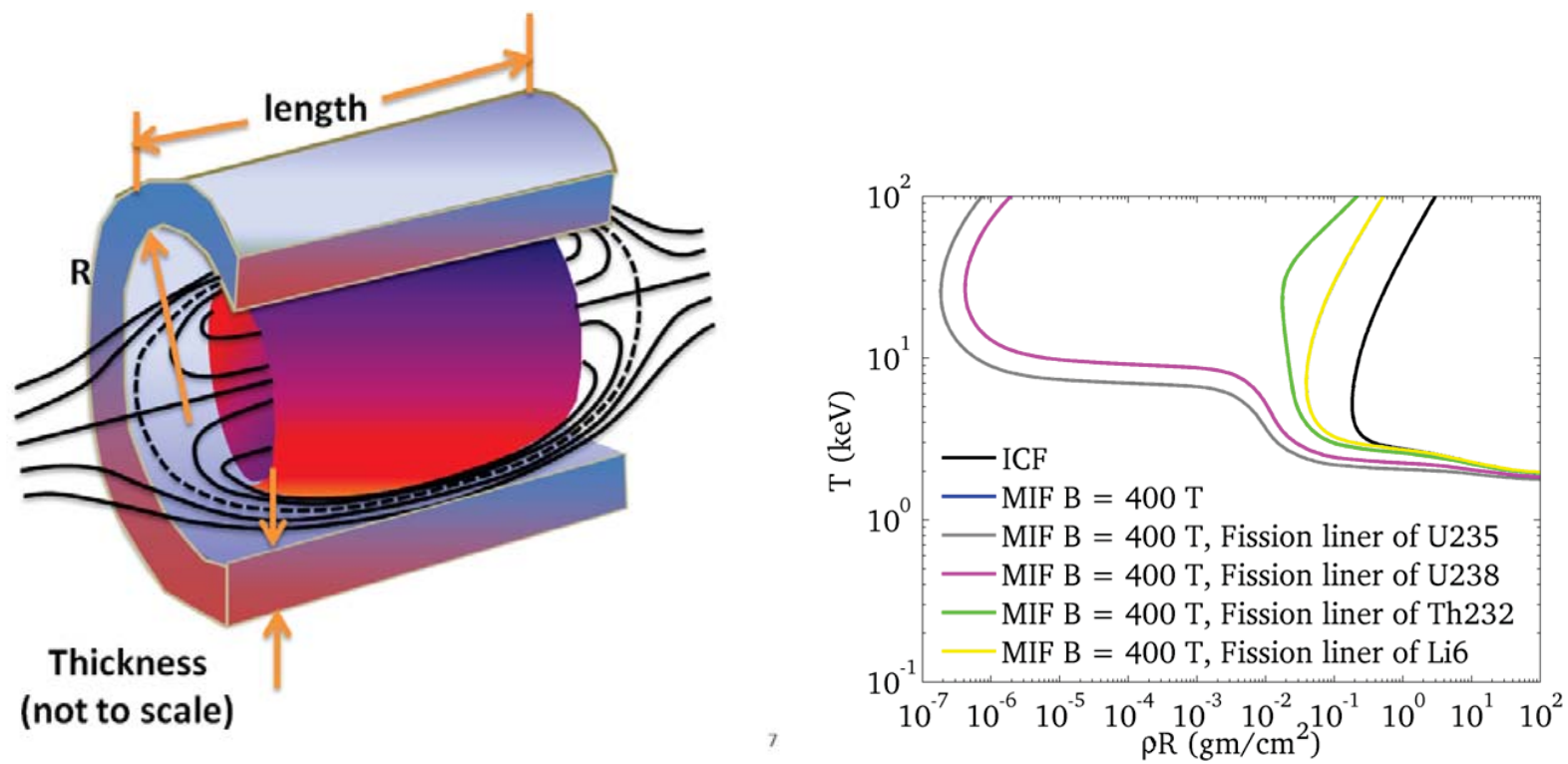

Figure 2. (a) Sketch of fission/fusion hybrid target. Magnetic field embedded in fusion target (center object), surrounded by a fissionable liner of Uranium, Thorium, or ${ }^{6} \mathrm{Li}$. (b) Contours for net power (fast fission + fusion) balancing against radiation and thermal conduction losses in $\rho R$ vs $T$ space, where $\rho$ is fusion target mass, $R$ is the fusion target radius, and temperature is the fusion target temperature.

The contour in Figure 2 shows the region in which the net power produced by nuclear reactions exceeds the loss mechanisms in $\rho \mathrm{R}$ vs $\mathrm{T}$ space. The black contour line is labeled 'ICF' and represents the typical region for net power production for inertial fusion. Generally, there is no embedded magnetic field. Beneath the contour ( 3 to $5 \mathrm{keV})$ the fusion power is not significant and the radiation and thermal conduction readily cool the target. The 'nose' on the left of the contour is primarily controlled by electron thermal conduction, which overtakes the fusion power at low densities. Above the contour, high temperatures lead to enhanced radiative cooling. When the target contains a magnetic field, the cross-field thermal conduction is suppressed, lowering the required $\rho \mathrm{R}$ and shifting the contour to the left. This result is highly dependent on the magnitude of the magnetic field. The effects of fission power are very apparent in this figure. For the cases investigated, we found that a target with radius $1 \mathrm{~mm}$ and surrounded by a $5 \mathrm{~mm}$ liner gave very promising results for both uranium isotopes. Specifically, the region of positive power production extended beyond the range of densities investigated. This is due to the relatively high fast fission cross sections for both of these isotopes, and the power is dominated by the fission power initiated by fast neutrons produced in the DT fusion reactions. The reason that the fission power is greater is that the energy per reaction is about 20 to 100 times greater ( $160 \mathrm{MeV}$ compared to a few $\mathrm{MeV}$ ) per reaction.

\section{SPFMax Development}

A smooth particle hydrodynamic code was completed and utilized for the Phase I effort. We leveraged development on another contract, but performed a significant amount of verification related to the present effort. SPH is a Lagrangian fluid code in which Euler, or Navier Stokes equations are solved on a discrete set of point masses which move at the local bulk velocity of the fluid. The primary advantage of the approach is the ability to accurately resolve fixed masses with distinct boundaries between gas and vacuum, and obviates the need for a computationally expensive grid for the space surrounding the system to be studied. Further, the SPH approach is naturally adaptive, and as a result, we have found that large, complex 3D problems can be modeled with just a few thousand particles and yet achieve accuracies within $10 \%$ of exact solutions for test cases.

The code we are developing is called Smoothed Particle Fluid with Maxwell equation solver (SPFMax). SPFMax is a hybrid between a smooth particle hydrodynamic plasma fluid solver and a 3D time dependent Maxwell equation solver utilizing the finite difference time domain method.

The partial differential equations are discretized using the smoothed particle formulation, which will be discussed below. We have developed the equations of motion and implementation strategy for the physics/chemistry model of 
electrically-controlled solid propellants (ESP). The flow solver is based on the following equations describing the motion of gases and ionized particles which will be present in the ESP experiments,

$$
\begin{aligned}
& \left(\frac{\partial}{\partial t}+\mathbf{u}_{\mathbf{e}} \cdot \nabla\right) n_{e}+\nabla \cdot \mathbf{u}_{\mathbf{e}}=0 \\
& \left(\frac{\partial}{\partial t}+\mathbf{u}_{\mathbf{i}} \cdot \nabla\right) n_{i}+\nabla \cdot \mathbf{u}_{\mathbf{i}}=0 \\
& n_{e} m_{e}\left(\frac{\partial}{\partial t}+\mathbf{u}_{\mathbf{e}} \cdot \nabla\right) \mathbf{u}_{\mathbf{e}}+\nabla p_{e}+e n_{e}\left(\mathbf{E}+\mathbf{u}_{\mathbf{e}} \times \mathbf{B}\right)=-\nabla \pi_{e}+\mathbf{R}_{\mathbf{e}} \\
& n_{i} m_{i}\left(\frac{\partial}{\partial t}+\mathbf{u}_{\mathbf{i}} \cdot \nabla\right) \mathbf{u}_{\mathbf{i}}+\nabla p_{i}-Z e n_{i}\left(\mathbf{E}+\mathbf{u}_{\mathbf{i}} \times \mathbf{B}\right)=-\nabla \pi_{i}-\mathbf{R}_{\mathbf{e}} \\
& \frac{3}{2} n_{e}\left(\frac{\partial}{\partial t}+\mathbf{u}_{\mathbf{e}} \cdot \nabla\right) k T_{e}+p_{e} \nabla \cdot \mathbf{u}_{\mathbf{e}}=-\pi_{e}: \nabla \mathbf{u}_{\mathbf{e}}-\nabla \mathbf{h}_{\mathbf{e}}-\left(\mathbf{u}_{\mathbf{e}}-\mathbf{u}_{\mathbf{i}}\right) \cdot \mathbf{R}_{\mathbf{e}}-Q_{i} \\
& \frac{3}{2} n_{i}\left(\frac{\partial}{\partial t}+\mathbf{u}_{\mathbf{i}} \cdot \nabla\right) k T_{i}+p_{i} \nabla \cdot \mathbf{u}_{\mathbf{i}}=-\pi_{i}: \nabla \mathbf{u}_{\mathbf{i}}-\nabla \mathbf{h}_{\mathbf{i}}-Q_{i}
\end{aligned}
$$

where the subscripts e and $i$ refer to electrons and ions, respectively, $\mathbf{u}$ is the velocity, $\mathrm{n}$ is the number density, $\mathrm{m}$ is the electron or ion mass, $\mathrm{p}$ is the pressure, $\mathbf{E}$ is the electric field, $\mathbf{B}$ is the magnetic field, $\mathrm{Z}$ is the ion charge, $\mathrm{k}$ is the Boltzmann's constant and $T$ is the temperature. The dissipative terms consist of the friction for $\mathbf{R}_{\mathrm{e}}$, viscous stress tensor $\pi$, thermal conduction $\mathbf{h}$, and the thermal equilibration term $\mathrm{Q}_{\mathrm{i}}$. Also note that the resistive dissipation term is $\left(\mathbf{u}_{\mathbf{e}}-\mathbf{u}_{\mathbf{i}}\right) \cdot \mathbf{R}_{\mathbf{e}}$. Expressions for the dissipation terms can be found, for example, in (Hans Goedbloed and Stefan Poedts 2004). Similar equations can be developed for neutrals.

One of the most significant technical challenges to such a model is accounting for electromagnetic field effects in propellant motion and chemistry. Most fluid codes using computational fluid dynamics (CFD) or magnetohydrodynamcs (MHD) are unable to include the required physics. Particle-in-cell (PIC) codes are potentially able to model ESP, however, they are unable to conduct parametric studies because each simulation requires prohibitively large computational resources. This hybrid electromagnetic/smooth particle hydrodynamic code approach simultaneously permits modeling all the required physics while being able to run 3D simulations in a few hours on a workstation.

Several methods can be employed to solve complex engineering problems. Numerical modeling techniques are useful when solving multidimensional partial differential equations that are too difficult to solve by hand. When it comes to fluids, numerical modeling generally explains behavior using Eulerian, Lagrangian, or combination models by means of fixed-point grids. However, grid-based simulations are not efficient or practical when solving problems involving highly deformable substances (Ala and Francomano, 2011). Problems are exacerbated when complex geometries and free spaces interact with deformable surfaces of fluids. Remeshing and additional mathematical processes may be required to produce an accurate model. These additions are extremely computationally taxing and time-consuming. When multiple fluids and processes are interacting with one another, meshes can no longer be used to illustrate the effects of the interactions. It becomes necessary to utilize mesh-less methods that account for conservation laws while accurately illustrating physical processes.

Mesh-less numerical models use a domain of arbitrarily placed nodes or particles to approximate solutions to partial differential equations (Ala and Francomano, 2011). Each particle carries the physical properties of a fluid or solid being tested. Particle interaction is observed over time in a purely Lagrangian sense which illustrates a more complete picture of overall behavior. Smoothed particle hydrodynamics (SPH) is a mathematical modeling technique that employs mesh-less strategies to solve complex differential equations. SPH approximates functions of physical properties by interpolating a continuous kernel over a domain of particle locations. The formula for the function approximation is 


$$
A_{a}(\boldsymbol{r})=\int A\left(\boldsymbol{r}^{\prime}\right) W\left(\left(\boldsymbol{r}-\boldsymbol{r}^{\prime}\right), h\right) d \boldsymbol{r}^{\prime}
$$

where $W$ is the kernel, $h$ is the smoothing length or the distance of influence from particle $a$, and $r$ is the position of the particle. The integral function can be numerically summed over a compact support domain of particles.

$$
A_{a}(r)=\sum_{b} m_{b} \frac{A_{b}}{\rho_{b}} W\left(r-r_{b}, h\right)
$$

For example, to calculate certain properties for particle $b$, the property being solved for, $A$, is divided by the density, $\rho$, and multiplied by the mass, $m$, and the kernel function. Accurate approximation depends primarily on the smoothing length, $h$. If the smoothing length is too small, interpolation of nearby particles would not be possible, or rather the simulation would not be able to detect the effects that nearby particles would normally have on one another. If the smoothing length is too large, then certain groups of particles would be seen affecting one another that normally would not (Ala and Francomano, 2011). Error must be minimized by discretizing the space around particles through differentiation of the property function.

$$
\nabla A_{a}(r)=\sum_{b} m_{b} \frac{A_{b}}{\rho_{b}} \nabla W\left(r-r_{b}, h\right)
$$

While grids inherently provide discretization for finite element models, the SPH kernel enables the space around particles to be discretized without the need for meshes, making SPH extremely versatile (Ala and Francomano, 2011). Further refinement of discretized space is possible through higher order differentiation which also reduces error at the cost of computation time. So to reiterate, property approximation depends completely on the smoothing kernel which, in turn, is a function of the smoothing length.

SPH was first developed in 1977 by Lucy, Monaghan, and Gingold as a method to reproduce equations of motion with more natural particles by means of kernel estimation techniques already in practice (Monaghan, 2005). Later development allowed SPH to automatically account for conservation laws, providing a path for utilization in most scenarios involving fluid dynamics, whereas, before, SPH could only be used to solve certain types of astrophysical problems (Monaghan, 2005). Today, SPH models are used extensively in a variety of fluids, elasticity, and fracture problems (Monaghan, 2005). It has even become a staple in the special effects industry for film and video games (Monaghan, 2005). Because of its versatility and accuracy, new adaptations of SPH are being created and tested every year to solve advanced problems in scientific arenas that have never experienced SPH treatment previously.

In engineering, multiple fluid interactions are extremely common, especially when chemical reactions are catalysts to electromechanical work. Propulsion sciences, especially, experience multiple fluid interactions on a regular basis. While simple numerical models help to show proof of concept, detailed chemistry and fluid models of combustion reactions have been difficult to produce with conventional methods. Furthermore, concurrently interacting physical processes have never been modeled in detail using consistent numerical methods. However, using SPH methodologies, it is possible to show particle interaction in the physical sense, not only between multiple fluids, but also between circuit-chemistry reactions. SPFMax is a program that uses SPH algorithms to simulate interacting fluids. New modifications to SPFMax have been able to produce smoothed particle electromagnetics (SPEM) models of circuits that illustrate current propagation through electrode solids. The circuit model is designed to interact with charged particles in a fluid - a truly novel concept. With SPFMax, several types of electric propulsion could potentially be modeled on a particle level. Additionally, with the added detail of the mesh-less Lagrangian methods employed by SPFMax, propulsion systems entering the conceptual stage could be modeled in detail and modified for maximum efficiency before small scale models are even built. The ultimate goal in development of SPFMax is to make novel and highly conceptual propulsion designs more accessible and cheaper to develop into fully integrated systems.

Early on in development of SPH, several non-hydrodynamic applications were explored, including modeling Maxwell's curl equations. This eventually led to the development of smoothed particle electromagnetics (SPEM). Like SPH, SPEM was created to solve complex problems without the need for meshes. In this manner, SPEM is a direct alternative to circuit modeling techniques like finite difference time domain methods. SPEM models solve Maxwell's equations over a domain of randomly spaced particles, just as SPH models are used to solve fluid dynamical equations. Maxwell's electric and magnetic field equations are 


$$
\begin{aligned}
& \nabla \times \mathbf{H}=\frac{\partial \mathbf{E}}{\partial \mathbf{t}}+\mathbf{J} \\
& \nabla \times \mathbf{E}=-\mu \frac{\partial \mathbf{H}}{\partial \mathbf{t}}
\end{aligned}
$$

where $\mathbf{H}$ represents the magnetic field, $\mathbf{E}$ - the electric field, $\mathbf{J}$ - the current density, and $\mu$ is the permeability of circuit material. Calculating changes in electric and magnetic fields is rather different from calculating physical properties such as temperature and density in that changes depend on field strengths of the neighboring particles. In other words, electric field changes depend on the strength of the magnetic field in neighboring particles, whereas magnetic field changes depend on the strength of the electric field in neighboring particles. This observation is more readily apparent in the expanded curl equations in the $x, y$, and $z$ directions, where new variables $\varepsilon, \sigma$, and $V$ represent the permittivity, conductivity, and volume of the circuit material respectively.

$$
\begin{aligned}
& \frac{\partial E_{x}}{\partial t}=\frac{1}{\varepsilon} \sum_{j=1}^{N}\left(H_{z}\left(r_{j}^{H}\right) W_{y}^{E}-H_{y}\left(r_{j}^{H}\right) W_{z}^{E}\right) V_{j}+\frac{\sigma E_{x}\left(r_{i}^{E}\right)}{\varepsilon}-\frac{J_{x}}{\varepsilon} \\
& \frac{\partial E_{y}}{\partial t}=\frac{1}{\varepsilon} \sum_{j=1}^{N}\left(H_{x}\left(r_{j}^{H}\right) W_{z}^{E}-H_{z}\left(r_{j}^{H}\right) W_{x}^{E}\right) V_{j}+\frac{\sigma E_{y}\left(r_{i}^{E}\right)}{\varepsilon}-\frac{J_{y}}{\varepsilon} \\
& \frac{\partial E_{z}}{\partial t}=\frac{1}{\varepsilon} \sum_{j=1}^{N}\left(H_{y}\left(r_{j}^{H}\right) W_{x}^{E}-H_{x}\left(r_{j}^{H}\right) W_{y}^{E}\right) V_{j}+\frac{\sigma E_{z}\left(r_{i}^{E}\right)}{\varepsilon}-\frac{J_{z}}{\varepsilon} \\
& \frac{\partial H_{x}}{\partial t}=\frac{-1}{\mu} \sum_{j=1}^{N}\left(E_{z}\left(r_{j}^{E}\right) W_{y}^{H}-E_{y}\left(r_{j}^{E}\right) W_{z}^{H}\right) V_{j} \\
& \frac{\partial H_{y}}{\partial t}=\frac{-1}{\mu} \sum_{j=1}^{N}\left(E_{x}\left(r_{j}^{E}\right) W_{z}^{H}-E_{z}\left(r_{j}^{E}\right) W_{x}^{H}\right) V_{j} \\
& \frac{\partial H_{z}}{\partial t}=\frac{-1}{\mu} \sum_{j=1}^{N}\left(E_{y}\left(r_{j}^{E}\right) W_{x}^{H}-E_{x}\left(r_{j}^{E}\right) W_{y}^{H}\right) V_{j}
\end{aligned}
$$

The expanded Maxwell's equations are based on formulations provided by Ala and Francomano with a slight difference in the additional current density term. This term becomes necessary to enable the SPFMax code to simulate interaction between the surface a solid electrode and the charged fluid particles of the fluid model (in this case, the fluid is a plasma). In the propulsion simulation, charged fluid particles take on current from the circuit model, essentially becoming part of it, while simultaneously encompassing the fluid model as well. Certain dynamics of the circuit-fluid model are very different from the circuit-electrode model. Because the electrode is a solid surface, the permittivity, permeability, and conductivity of the circuit material is assumed constant. However, those same properties must be interpolated in the plasma because the plasma is deforming and accelerating over the course of the simulation. As particle concentration changes, physical properties change.

The magnetic nozzle, such as shown below in Figure 3, is needed for our propulsion concept because the high temperatures reached by the plasma will cause rapid erosion and failure of material nozzles, and even if those limitations can be overcome by controlled ablation off the wall, significant weight penalties will be incurred by the additional radiative cooling needed. Towards the goal of studying pulsed magnetic nozzles, we developed a series of verification tests to provide confidence in the numerical output of the gasdynamic physics of SPFMax, and proceeded to study the effects of pulsed gas nozzles (see Figure 4). The purpose was to take a first step in understanding some of the basic physics of expansion from initial hot, cylindrical targets into directed thrust. 


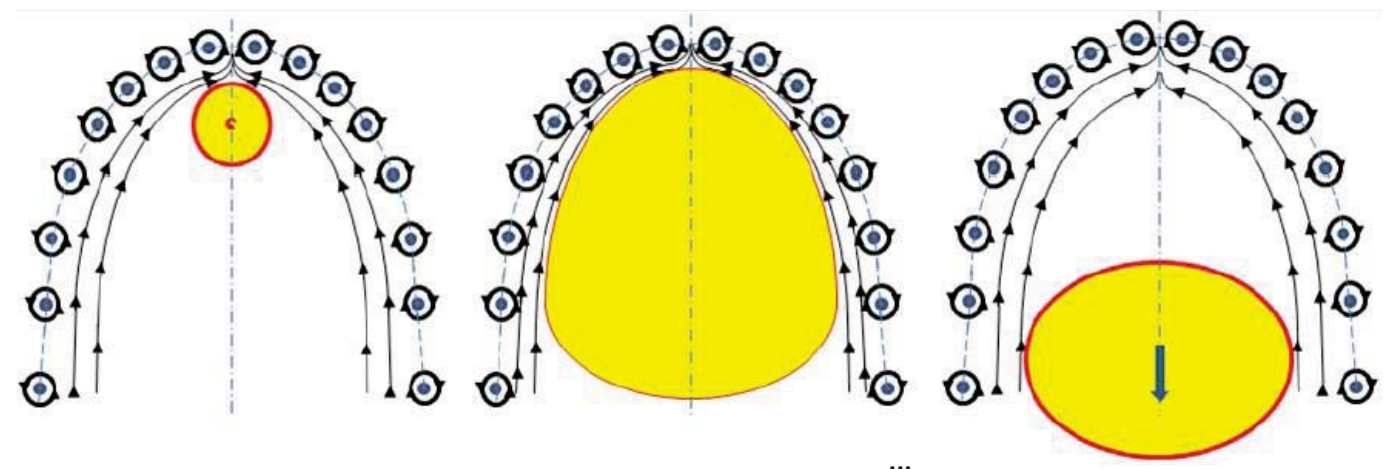

Figure 3. Pulsed magnetic nozzle operation (Ref. ${ }^{\text {iii)}) . ~}$

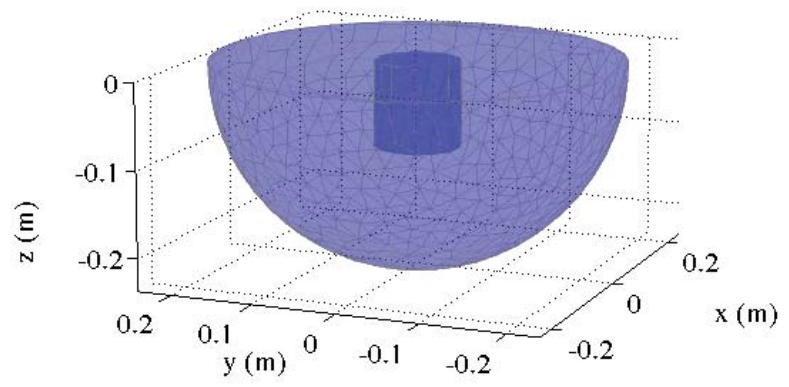

\section{Figure 4. SPFMax model of a solid state gas nozzle.}

A shock tube model was studied that allows for testing and debugging of key physics for flows commonly found in nozzles, including a mixture of subsonic and supersonic flows, shock waves, and thermal expansion. We demonstrated convergence as we increased particle resolution, and the average error for 10,000 particles (particle scale length of $\sim .1$ to $1 \mathrm{~cm}$ ) was within $5 \%$. We note that higher accuracy can be achieved by increasing the number of particles, but settled on this problem size since $3 \mathrm{D}$ simulations could be accomplished in a few minutes for most cases.
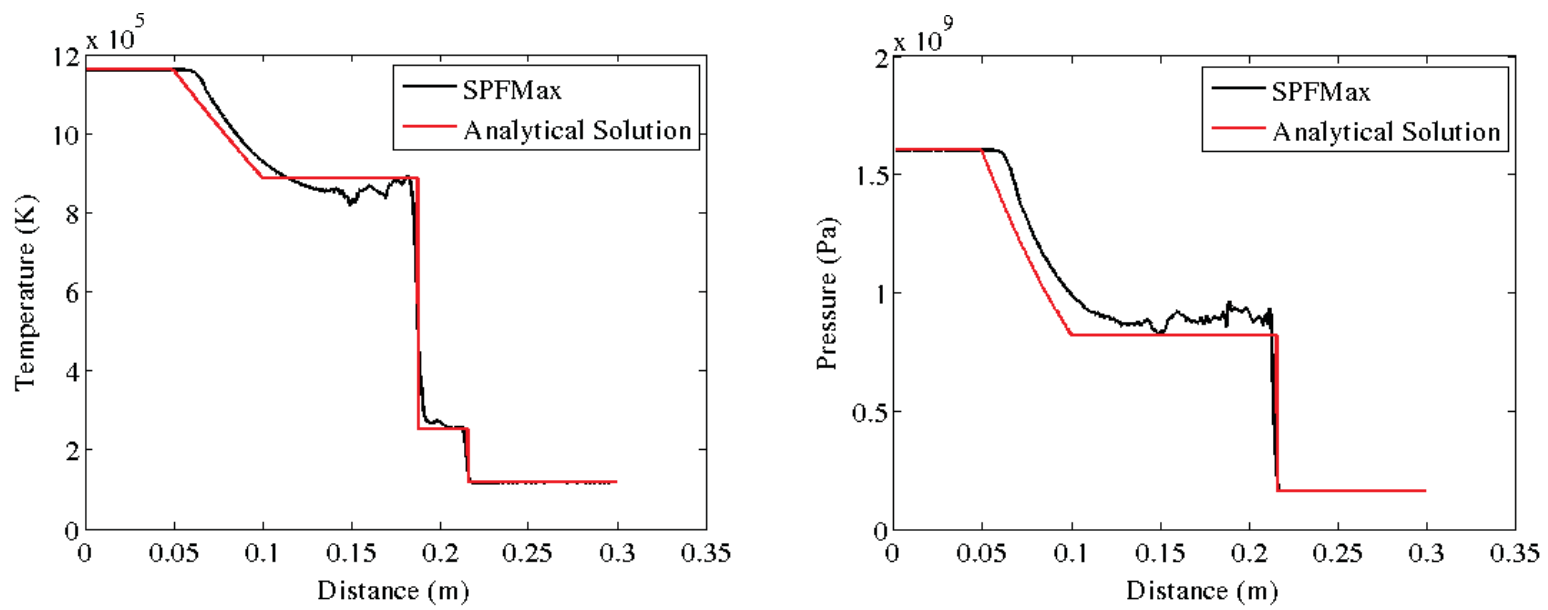

Figure 5. Temperature and pressure in a shock tube with temperatures between 10 and $100 \mathrm{eV}$. 
We then ran a spherical gas expansion test. For this model, we expect that the particles should expand isotropically with a mean expansion velocity approaching $u_{\max }=\sqrt{2 \mathrm{C}_{\mathrm{v}} \mathrm{T}_{0}}$. A sequence of three particle scatter plots shows that there are not anomalous motions and the particle expansion behaves as it should. Figure 7 shows that the mean expansion velocity does indeed approach the predicted result, which is an indication that isentropic expansion is modeled accurately and that energy is conserved in the time integration algorithm.
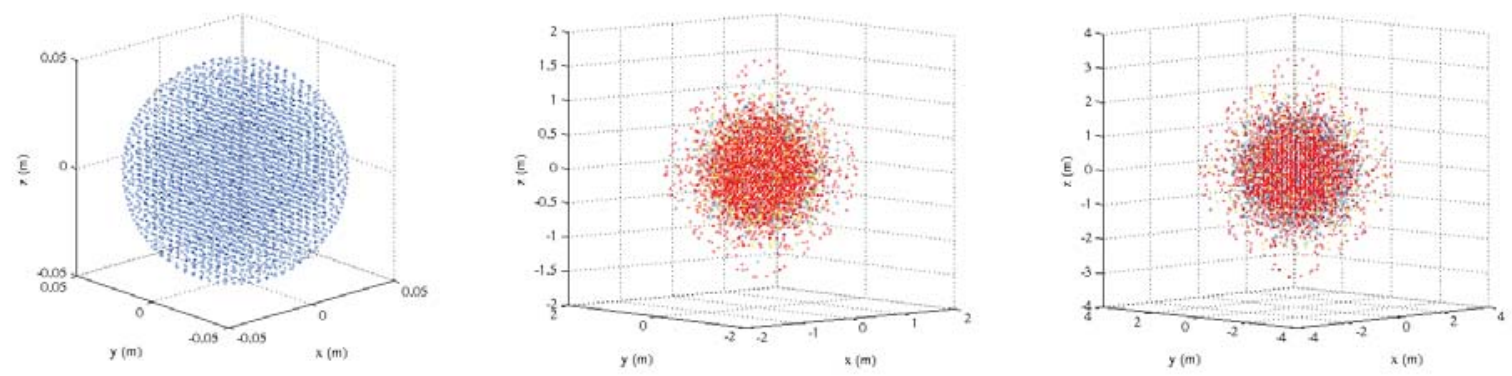

Figure 6. Spherical gas expansion at 0,10 , and $20 \mu \mathrm{s}$.

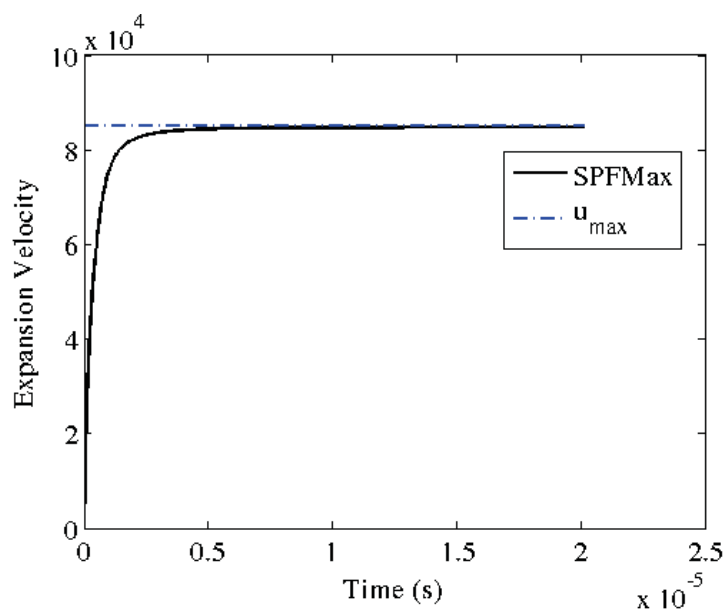

Figure 7. Mean expansion velocity for spherical expansion test.

For the pulsed gas nozzle tests, we explored over a hundred different cases involving effects of initial nozzle radius and relative position, size, and length to radius ratio of a cylindrical gas target, as well as effects of initial density and temperature. We found that 'pancake' like initial targets with radii matching the inner nozzle radius at the exit, placed near the nozzle exit, gave the highest propulsion efficiencies (directed kinetic energy divided by initial thermal energy). Figure 8 shows the initial conditions for such a nozzle along with late time expansion once most of the mass has exited. There is some divergence in the plume, which contributes to the $30 \%$ or so energy not converted to directed kinetic energy. In this example, the efficiency approaches $70 \%$ with an Isp of $6500 \mathrm{~s}$.
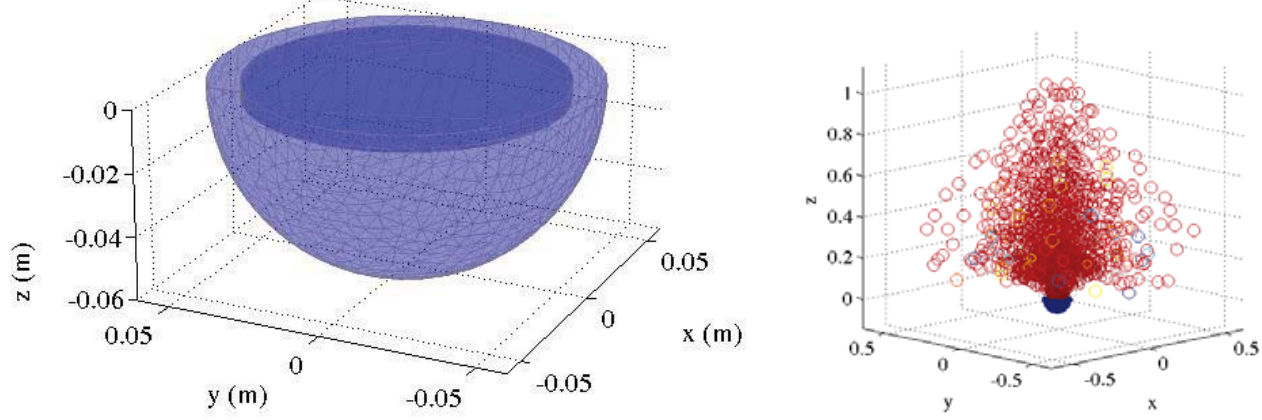

Figure 8. Initial conditions and late time expansion phase for one of the high efficiency nozzle configurations. 

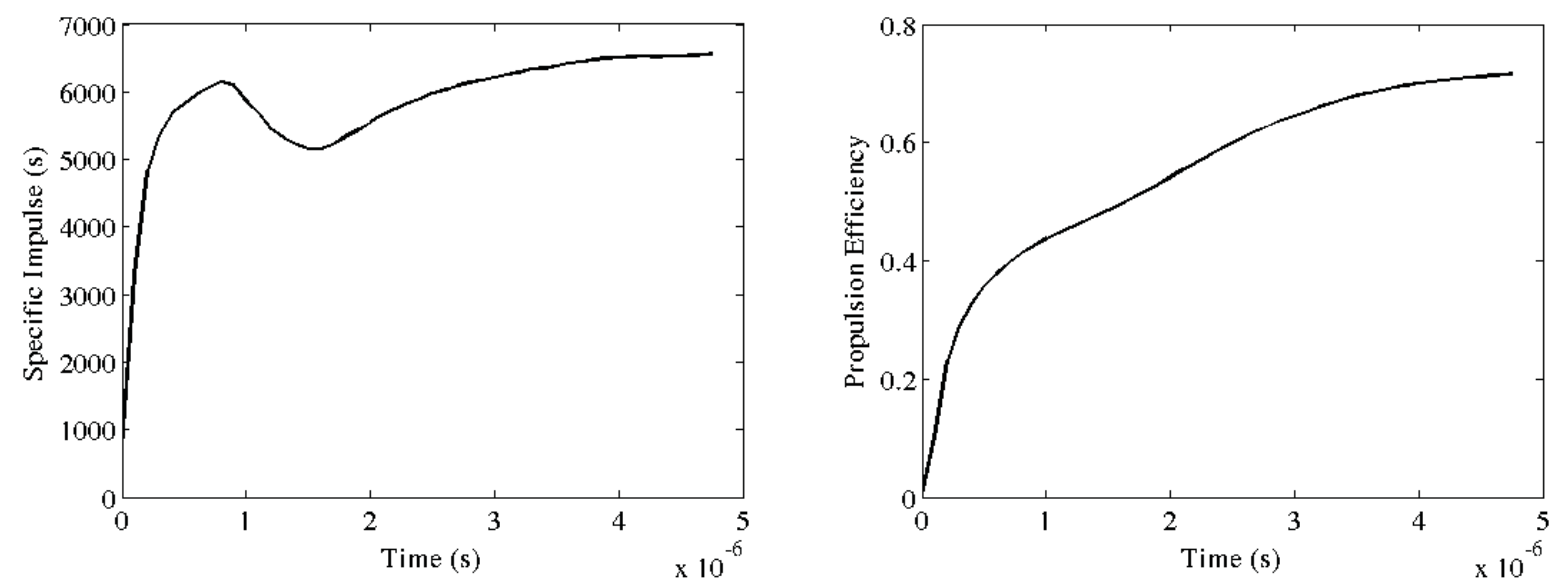

Figure 9. Specific impulse and propulsion efficiency vs. time. Note the specific impulse dips around 1 as while the gas 'bounces' off the wall. The run is stopped once $95 \%$ of the mass has exited the nozzle.

We anticipate that the physics of expansion in more realistic cases with a magnetic nozzle are going to be somewhat different. For example, the plasma will compress the magnetic field, and perturbations at the vacuum field/plasma interface may cause instabilities. The targets will likely have strong gradients which will modify the expansion behavior. Finally, finite resistivity and other diffusive processes will trap plasma on the field. As we develop the code further we will explore these effects hopefully in parallel with an experimental program. We view the gas nozzle results as a point of reference for idealized behavior, and in doing so this may inform designers on how to properly design targets integrated into magnetic nozzles.

\section{Mission Performance}

A proper assessment of propulsion system performance can only be obtained by incorporating the system into a vehicle design and then flying it on a number of realistic missions. To assist in this effort a simple mass estimation tool has been developed to enable the PuFF team to 'build' a range of vehicles as needed to support the performance evaluation effort.

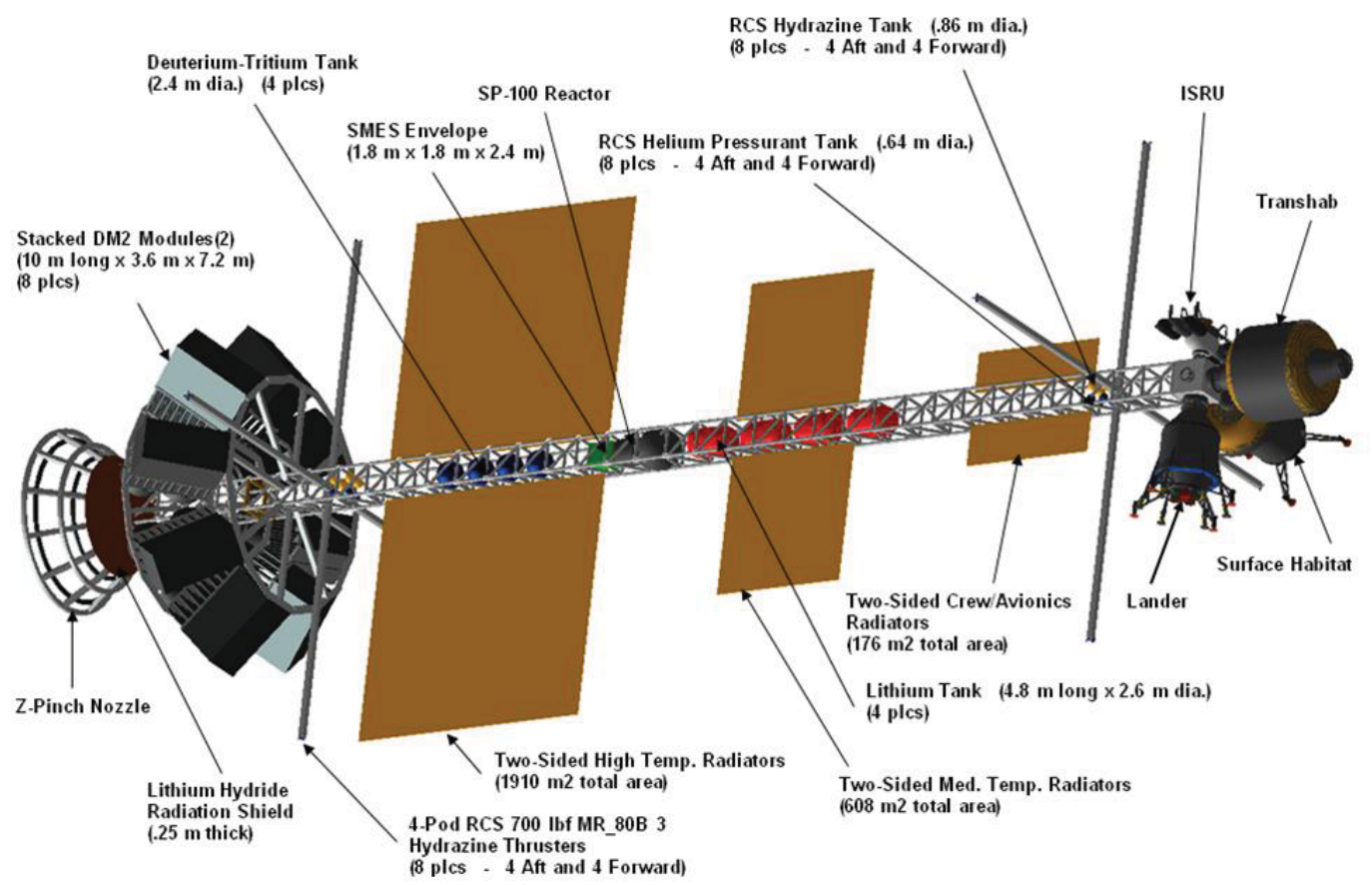

Figure 10. Vehicle Configuration for Mass Estimation Tool

American Institute of Aeronautics and Astronautics 
This model is based on a vehicle study that was conducted in support of a previous Z-Pinch propulsion concept study (Ref. ${ }^{\text {iv }}$ ). The vehicle, which is shown below, was designed to conduct several missions in the inner solar system, including a crewed Mars mission, with 90-day (each-way) interplanetary transit times. It forms a good basis for evaluating PuFF performance because most of its major components are of the same type as those that will be required on a PuFF vehicle (e.g. tanks for both D-T and lithium). The only minor exception is the uranium storage and supply system that will be required for PuFF.

The major vehicle components and their approximate scaling rules are as follows.

\begin{tabular}{|l|l|}
\hline Vehicle Component: & Mass Scaling Rule: \\
\hline Magnetic Nozzle & Linear with energy generated per pulse \\
\hline Radiation Shielding & Linear with energy generated per pulse \\
\hline D-T Tanks & Linear with total mass of D-T mixture carried \\
\hline Lithium Tanks & Linear with mass of lithium carried \\
\hline Main Vehicle Truss & Linear with vehicle wet mass and vehicle length \\
\hline Other Primary Structures & Linear with vehicle wet mass and vehicle length \\
\hline Secondary Structures & 20\% of 'Other Primary Structures' mass \\
\hline Capacitor Banks & Linear with energy input required per pulse \\
\hline Marx Generator Circuitry & $6 \%$ of 'Capacitor Banks' mass \\
\hline Reaction Control System & Linear with vehicle total wet mass \\
\hline Low Temperature Heat Rejection System & Linear with low temperature heat rejection rate \\
\hline Medium Temperature Heat Rejection System & Linear with medium temperature heat rejection rate \\
\hline High Temperature Heat Rejection System & Linear with high temperature heat rejection rate \\
\hline LN2 Seed Coil Cooling System & Linear with energy generated per pulse \\
\hline $\begin{array}{l}\text { Vehicle Power System } \\
\text { Based on SP-100 reactor and associated } \\
\text { power distribution equipment }\end{array}$ & $\begin{array}{l}\text { Linear with maximum electrical power requirement for } \\
\text { the vehicle }\end{array}$ \\
\hline Avionics System & Constant \\
\hline
\end{tabular}

It must be emphasized that the model is only intended to provide an approximate vehicle mass. It will be noted that there is no mass margin allowance illustrated above. A 30\% 'mass growth allowance' was used in the original source study, use of the approximate scaling rules above will yield an approximate inert mass margin based on the $30 \%$

An example of the model output is given below in Figure 21, where the vehicle dry mass is shown as a function of the medium temperature heat rejection rate. Curves are given for a range of high temperature heat rejection rates (from 100 to $600 \mathrm{MW}$ ). And clearly demonstrate the importance of rejecting as much of the total heat load as possible at high temperature.

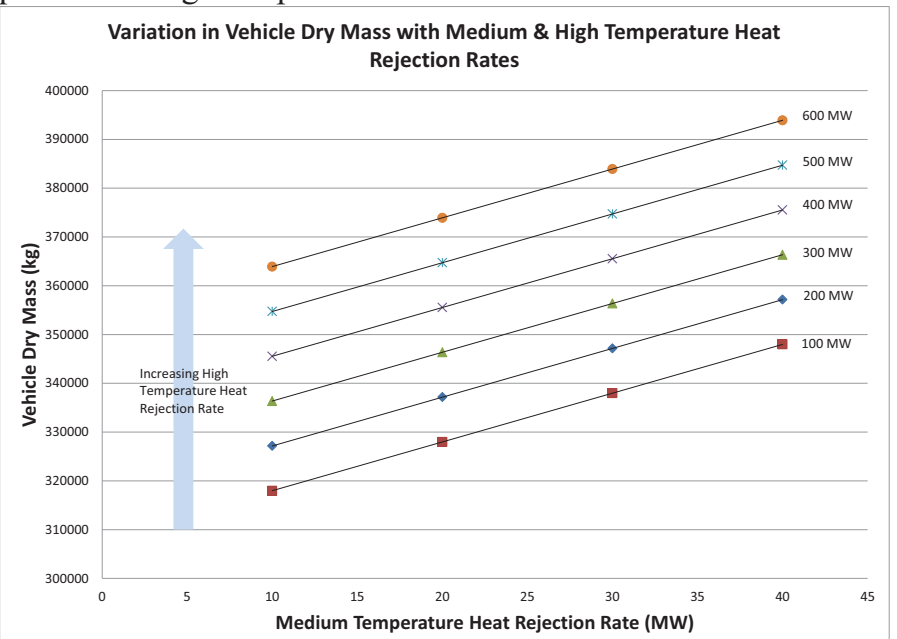

Figure 11. Sample Output from Vehicle Mass Estimation Tool

American Institute of Aeronautics and Astronautics 
Using the point design we resized the vehicle shown in Figure 10 for a couple of new missions. The first mission was a sprint to Mars, carrying crew or payload to support a Martian outpost. The engine was set by the point design, and the missions shown in Figure 12 represent our best effort to optimize performance around the point design. For instance we determined that the point design engine can carry a $25 \mathrm{mT}$ capsule from Earth to Mars in 37 days. The Initial Mass in Low Earth Orbit for this concept is $190 \mathrm{mT}$, as shown in the table below. This mass could be lofted to orbit using a Block I and a Block II Space Launch System (SLS)

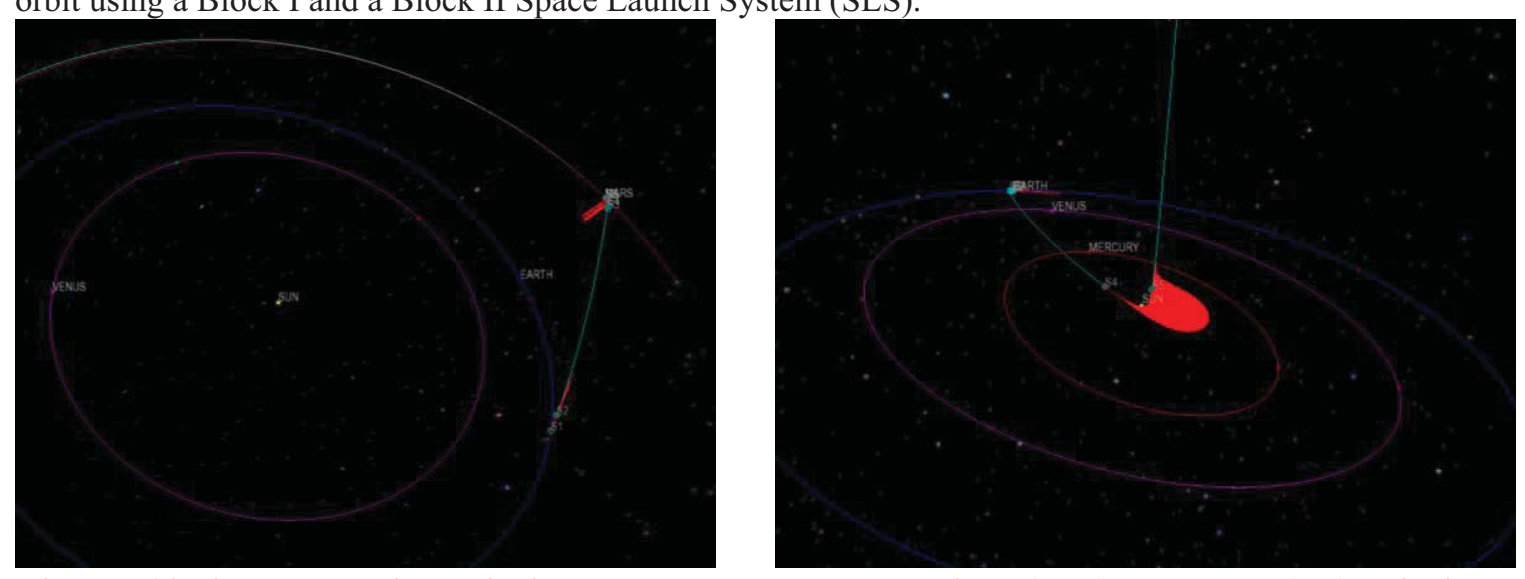

Figure 12. 37 day sprint mission to Mars and a robotic TAU (thousand AU) mission

Table 1. Mass Breakdown for Mars Direct and TAU mission concepts

\begin{tabular}{|l|l|l|}
\hline Subsystem & Mars Express & TAU Mission \\
\hline Magnetic Nozzle & $14.83 \mathrm{mT}$ & $14.83 \mathrm{mT}$ \\
\hline Radiation Shielding & 14.01 & 14.01 \\
\hline D-T Tankage & 5.0 & 5.0 \\
\hline Li Tankage & 1.45 & 0.94 \\
\hline Truss & 2.71 & 2.09 \\
\hline Other Primary Structures & 0.64 & 0.49 \\
\hline Secondary Structures & 0.13 & 0.10 \\
\hline Capacitor Banks & 2.10 & 2.10 \\
\hline Marx Generator Circuitry & 0.13 & 0.13 \\
\hline RCS Wet Mass & 1.03 & 0.79 \\
\hline Low Temp Heat Rejection & 1.30 & 1.30 \\
\hline Medium Temp Heat Rejection & 14.82 & 14.82 \\
\hline High Temp Heat Rejection & 1.26 & 1.26 \\
\hline LN2 Seed Coil Cooling & 8.41 & 8.41 \\
\hline Auxiliary Power & 4.40 & 4.40 \\
\hline Avionics & 0.39 & 0.39 \\
\hline Payload & 25.00 & 10.00 \\
\hline Dry Mass (without MGL) & $\mathbf{9 4 . 8 7}$ & $\mathbf{7 7 . 5 7}$ \\
\hline Mass Growth Allowance (30\%) & 40.66 & 35.21 \\
\hline Total Dry Mass & $\mathbf{1 3 5 . 5 3}$ & $\mathbf{1 1 2 . 7 8}$ \\
\hline Fuel & 56.02 & 37.03 \\
\hline Total Wet Mass & $\mathbf{1 9 1 . 5 5}$ & $\mathbf{1 4 9 . 8 1}$ \\
\hline & &
\end{tabular}

We also looked at the same point design for a robotic explorer to the outer solar system. Here we designed a vehicle that starts out at LEO at $150 \mathrm{mT}$ and is capable of delivering $10 \mathrm{mT}$ of payload to interstellar space. The terminal 
velocity of the vehicle is $0.075 \mathrm{AU} /$ day translating to passing the termination shock in 5 years, a distance of $275 \mathrm{AU}$ in 10 years, reaching the solar gravitational lens in 20 years and achieving 1000 AU (0.016 ly) in 36 years. Such a mission could be completed in the lifetime of a single researcher, providing a wealth of information about local interstellar space and our own solar system's interaction with it.

\section{Experimental Efforts}

The PuFF team is assembling, refurbishing or repurposing 5 different pulser systems. These systems operate at varying power levels $200 \mathrm{~J}, 1 \mathrm{~kJ}, 8-10 \mathrm{~kJ}, 62 \mathrm{~kJ}, 576 \mathrm{~kJ}$. With a successful Phase II award the team will test at these varying power levels z-pinch processes to drive out the progression of plasma instabilities in a z-pinch process. These results will be combined with the SPFMax analysis above to build a predictive model. The benchmarked model will assist in developing the exact specifications for a PuFF propulsion system that maximizes performance while minimizing mass.

The efforts to set up this wide array of pulser units is deserving of a paper in its own right. However included below is a test run of a $1 / 64$ " diameter $\mathrm{Li}$ wire that was detonated in the 8-10 kJ pulser system, known at MSFC as the Micrometeoroid Gun (MMG). As can be seen from the streak camera there is a rapid change in the wire from one frame to the next during the pinch process.

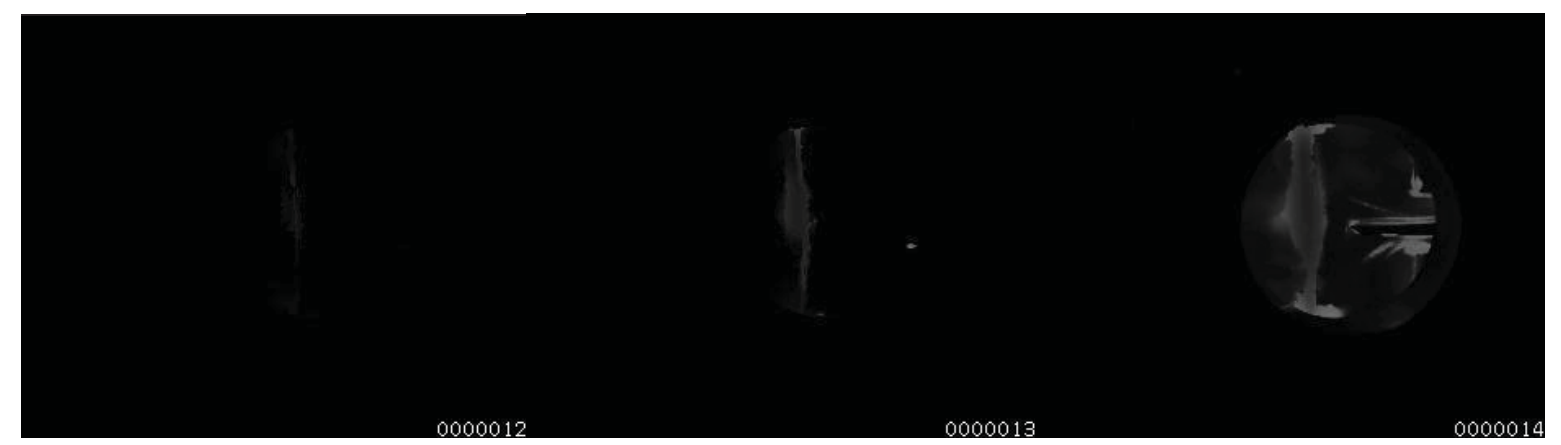

Figure 13. Test fire of 1/64 in Li wire at $8 \mathrm{~kJ}, 22 \mathrm{kV}, 320 \mathrm{kA}$. Frame 1 (12 microseconds) shows ends of wire starting to glow. Frame $2(13 \mathrm{~ms})$ shows illumination along wire and some spalling. Frame 3 (14 ms) demonstrates wire implosion and subsequent expansion.

\section{Conclusion}

As to be expected for a NIAC Phase I PuFF is a work in progress. However our steady state results illustrate great potential for breakeven energy production and beyond. A non-optimized application of a PuFF engine point design demonstrates game changing capabilities in two missions with expectations of higher reductions from further optimization and inclusion of LTD's. A bulleted list of our accomplishments includes:

Theory

- Verified 3D code on shock capturing and expansion

- Studied pulsed nozzle efficiency

- electromagnetic plasma code nearly complete

- Hybrid Fission/fusion power balance showing much lower energies required for breakeven Experiments

- Built a complementary team of collaborators prepared to conduct wire array and gas puff z-pinch experiments

- developing and configuring five pulsed power systems with energy levels spanning five orders of magnitude in capacitor bank energy to develop scaling laws for advanced propulsion design

$\underline{\text { Systems Analysis }}$

- Crewed Mars vehicle delivers $25 \mathrm{mT}$ of capsule, crew to Mars in 37 days starting with a $190 \mathrm{mT}$ vehicle in LEO. Suitable for a Block I and Block II SLS

- Interstellar probe capable of delivering $10 \mathrm{mT}$ to $1000 \mathrm{AU}$ in 36 years, would pass through the termination shock in 5 years. IMLEO is $150 \mathrm{mT}$, suitable for a Block II SLS and commercial launch vehicle 


\section{Acknowledgments}

The PuFF team thanks NIAC for the opportunity to conduct this research. This effort has been very exciting for us, and it would not have come to fruition without their support. Our efforts to make pulsed fission-fusion a reality has been supported by a number of other people. Jim Martin, Branch Chief of ER24/Propulsion Research and Technology Branch, and Boise Pearson, Team Lead in that branch, have supported our efforts without fail. Whenever we needed a new fitting or help finding a subject matter expert they were willing to make the connection. Andrew Keys, Ph.D., Chief Technologist at MSFC and Dale Thomas, Ph.D., MSFC Associate Center Director, have advised this team and provided managerial guidance on a number of occasions. Bill Emrich, Ph.D. gave us valuable insights into the fission process for very short compression timelines. Richard Eskridge was invaluable in helping us work with the Micro-Meteoroid Gun, which he developed, and other insights into experimental high power systems and plasma processes.

\section{References}

'Dyson, George. Project Orion - The Atomic Spaceship 1957-1965. Penguin. ISBN 0-14-027732-3

ii $h$ ttp://www.nndc.bnl.gov/exfor/endf00.jsp

iii 'Conceptual Design of In-Space Vehicles for Human Exploration of the Outer Planets', Adams, R.B et al, NASA/TP-2003-212691.

iv 'Z-Pinch/Dense Plasma Focus Thermonuclear Propulsion System', by the Advanced Concepts Office (ED04), Marshall Space Flight Center, October 8, 2010. 